\title{
Optimal Sizing and Sitting of Distributed Generations in Power Distribution Networks Using Firefly Algorithm
}

\author{
Aref Jalili $^{1} \cdot$ Bahman Taheri ${ }^{2}$ \\ Received: 23 April 2018 / Accepted: 4 March 2020 / Published online: 26 March 2020 \\ (C) Springer Nature Singapore Pte Ltd. 2020
}

\begin{abstract}
Compared to centralized generation, distributed generations (DGs) have numerous advantages including real power loss reduction, voltage deviation reduction, network stability enhancement, emission reduction, capacity increase of transmission lines and congestion reduction in distribution networks. Optimum placement of DGs plays a crucial role in this regard. In this paper, Firefly Algorithm (FA) was employed for sizing / sitting of various DGs in distribution networks. The aim of this paper was to minimize power loss by taking into account power factor, active power, and reactive power of DGs. Furthermore, different active and/or reactive generating/consuming DGs were also considered. The performance analysis of the proposed method was validated on standard IEEE 33- and 69-bus test systems.
\end{abstract}

Keywords Distribution network · Distributed generation $\cdot$ Power loss reduction $\cdot$ Firefly algorithm

\section{Introduction}

In recent decades, it was well proved that distributed generations (DGs) is a viable, environmentally-friendly solution to reduce emissions and power loss reduction as they are mostly situated near the load centers. Although DGs have several environmental and economical benefits, they may impose several operational issues to distribution systems, if placed illogically. These may include but are not limited to relay coordination problems caused by reverse power flow, voltage rise issues, power quality and voltage stability issues, and among others. In this respect, teaching learning-based optimization (TLBO) algorithm reported in [1] is a parameter independent intelligent technique which was developed and subsequently used for the optimal placement of energy resources in distribution systems. Even though TLBO is parameter independent and has a very fast convergence rates, it is prone to be trapped locally in maxima/minima. It is observed that TLBO often converges to local minima when the number of DGs and/or

Bahman Taheri

b.taheri@iauardabil.ac.ir

1 Department of Electrical Engineering, Ardabil Branch, Islamic Azad University, Ardabil, Iran

2 Young Researchers and Elite Club, Ardabil Branch, Islamic Azad University, Ardabil, Iran operating constraints in the distribution system increase. Progress in soft computing methods have led to the development of a lot of evolutionary optimization algorithms for the optimum placement of DGs in distribution systems. Some of the widely used techniques in engineering applications are genetic algorithms (GA), artificial bee colony (ABC) algorithm, particle swarm optimization (PSO) algorithm, ant colony optimization (ACO) algorithm, bacterial foraging optimization (BFO), and among others. Some comprehensive research works on the employment of GA for optimum placement of DGs in distribution networks have been previously reported. In [2], various methods have been suggested for locating different types of DGs. Position and capacity of DGs are mostly determined with the goal of power loss minimization in distribution networks. Optimal power factor for DG supply along with active and reactive powers are specified in this research. In addition, various types of DGs injecting active and reactive powers into different distribution buses are considered in the proposed method. Ref. [3] details the ant lion optimization algorithm (ALOA) for optimum allocation and sizing DG-based renewable sources for radial distribution systems. Authors in [4] discuss placement of various DG units in order to get the maximum power loss reduction in large distribution networks. In [5], a novel method is proposed for deployment of DG units on radial distribution feeder with heavy loading and non-uniform distributed load. Most of current algorithms for distribution feeders with uniform 
distributed load are designed only with one DG. The suggested approach in this work is able to operate in distributed load condition in random fashion with low power factor for several DGs. In [6], an optimized method was suggested to get appropriate DG location based on cost on value analysis. This method considers economic and technical factors such as energy loss, reliability indices of load points as well as costs of DG, especially, its portability. The proposed method was implemented on a test network and the effects of various parameters such as load growth rate and load forecast uncertainty (LFU) on optimum place of DG are fully studied. Ref. [7] provides directions for the studies in the problem of optimum DG placement or intending to do research in this area. In [8], the effectiveness of GA and PSO for optimal placement and sizing of DG in the radial distribution system is discussed. In [9], multi-objective formulation to determine DG size and site in distribution network is suggested. This method is adapted in order to use designer opinion on making-decision about tradeoff among various purposes of network development and upgrade, power loss cost, energy not supplied (ENS) cost, and the cost for customers who should be supplied. In [10], referring to necessity of DGs due to load demand growth, getting place and size of these resources is considered as an influencing factor in distribution networks' power loss reduction. Ref. [11] proposes a novel method based on PSO in order to reduce power loss by DG placement. Authors in [12] discuss the possibility of installing various DG types in different places in terms of geographic and environmental aspects regarding DGs' share in producing power on distribution level. In this research, the combination of various DGs and their effect on power loss is discussed using PSO and adaptive PSO (APSO). In [13], a solution is proposed for distribution network planning using DGs. In this work, three main factors related to multiple DGs placement are detailed by a multi-objective method. The factors include voltage stability, power loss, and network voltage variations. The optimum placement of DGs is solved by firefly algorithm (FA) in order to reduce distribution network power loss and improve voltage profile. As mentioned earlier the aims of this research is power loss minimization and voltage stability improvement considering operational and protective constraints of the radial distribution networks. Performance analysis of this work is performed by running on two scenarios on IEEE 33-69 bus standard test system. In addition, the results obtained are compared with the previously published valid works.

\section{Problem Formulation}

Power flow tool investigates the steady state analysis of an interconnected power system under normal operating conditions. In general, conventional power flow programs in distribution networks are divided into three methods: (i) Newton-Raphson-based techniques, (ii) Gaussian $Z_{\text {bus }}$-based methods and (iii) backwardforward sweep approaches [13]. The principals of Gaussian $Z_{\text {bus }}$-based methods are comprehensively reported in [14]. This method uses Sparse-Admittance matrix and equal current injections to solve network equations. Backward-forward sweep methods have been widely used due to high speed and less computer memory requirement as well as their overall great features in distribution system calculation [15]. Here, the general algorithm has two main steps of backward sweep and forward sweep. This algorithm iterates until convergence is achieved. These methods are divided into three classes: (i) current summation method, (ii) power summation method, and (iii) impedance summation method.

Overall steps involved in backward-forward sweep algorithm are as follows:

\section{Backward-Sweep}

In this step, the objective is to calculate the current flowing through network branches. First, required load current connected to $i$ th node is obtained by an initial voltage guess for first iteration as 1 p.u. (Eq. (1)):

$\mathrm{I}_{\mathrm{i}}=\left(\frac{\mathrm{P}_{\mathrm{i}}+\mathrm{Q}_{\mathrm{i}}}{\mathrm{V}_{\mathrm{i}}}\right)^{*}$

where $P_{i}, Q_{i}$, and $V_{I}$ are respectively active power, reactive power and voltage of $i$ th bus.

By starting from terminate buses and adding load currents and, then, moving toward slack bus, branch currents are obtained by:

$J_{n}=I_{i}+\sum_{m \in M} J_{m}$

where $J_{n}$ denotes $n$th branch current, $i$ denotes terminate node of branch $n, I_{i}$ denotes injected current into the load connected to the $i$ th node, $M$ denotes a set of branches connected to branch $n$ in $i$ th node and $I_{m}$ denotes $m$ th branch current.

\section{Forward Sweep}

In this step, the goal is to calculate network bus voltages with slack bus voltage of 1 p.u. By starting from the buses connected to slack bus and moving toward end branches, voltage of receiving bus of $n$th branch (i.e. $i$ ) Iis calculated by considering currents obtained in previous step:

$V^{i}=V^{j}-Z_{n} J_{n}$

Fig. 1 depicts backward and forward sweeps for achieving branch currents and bus voltages. 


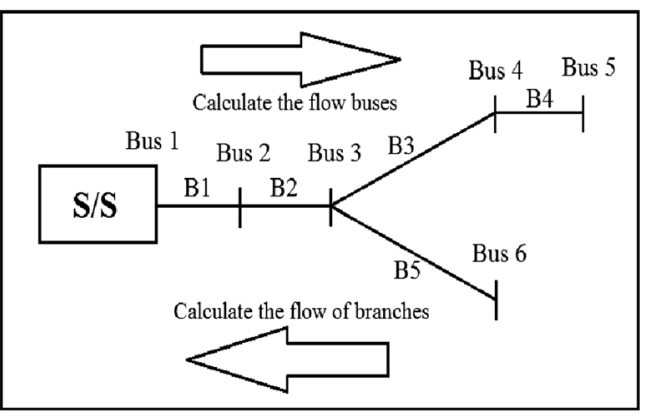

Fig 1. Backward and forward sweeps for a hypothetical network [16].

\section{Voltage Non-adaptability Calculation}

The goal of conducting power flow is to calculate bus voltage phase and amplitude. Obviously, by calculating bus voltages, the other required parameters such as power loss, line current, etc. can be also calculated. Once one step of backwardforward process is done, voltage is obtained in new iteration. And, the voltage non-adaptation for all buses is obtained by:

$\Delta V^{i(k+1)}=\left|V^{i(k+1)}\right|-\left|V^{i(k)}\right|$

where $k$ is the number of iterations. If any values of $\Delta V^{i}$ is more than convergence criterion, power flow process will be also iterated in order to satisfy convergence criterion by:

$\max \left(\left|\Delta V^{(k+1)}\right|\right)<\varepsilon$

The maximum voltage difference calculated for each bus in $(k+1)$ th iteration with respect to the previous iteration should satisfy convergence criterion; otherwise, these steps should continue until satisfying the above-mentioned condition.



Fig 2. FA flowchart [17].

\section{Distributed Generation Modeling}

DGs in distribution networks are divided into three groups in terms of their operation. Various operational modes of DG units are:

- DG utilization in parallel structure with the feeder;

- DG utilization with fixed power factor;

- DG utilization with fixed voltage.

In first two modes of power flow algorithm, DG units are considered as PQ buses; while, in the last one, generating buses are in PV state.

In this paper, DG units are modeled in four types considering the technology type and all of them are used as PQ model, or active and reactive power generation or consumption models.

- Type-1: Solar cells and fuel cells as the sole active power generator;

- Type-2: synchronous compensators as the sole reactive power producer;

- Type-3: synchronous generators as active and reactive power producer;

- Type-4: asynchronous generators of wind turbine type as active power producer and reactive power consumer.

\section{Proposed Method: Firefly Algorithm (FA)}

The Firefly Algorithm (FA) was developed by Yang in 2008 based on the idealized behavior of the flashing characteristics of fireflies. For simplicity, these flashing characteristics may be idealized as the following three rules:

(1) All fireflies are unisex so that one firefly is attracted by the other ones regardless of their sex;

(2) Attractiveness is highly proportional to the brightness, hence, for any two flashing fireflies, the less brighter one moves towards the brighter one and their brightness decreases with the increase in their distance. If no one is brighter than a particular firefly, it will move in random manner;

(3) The brightness or light intensity of a firefly is influenced or determined by the landscape of the objective function to be optimized.

\section{Steps to Implement FA}

FA is implemented through the following steps: 
Fig. 3 Standard IEEE 33-bus test system

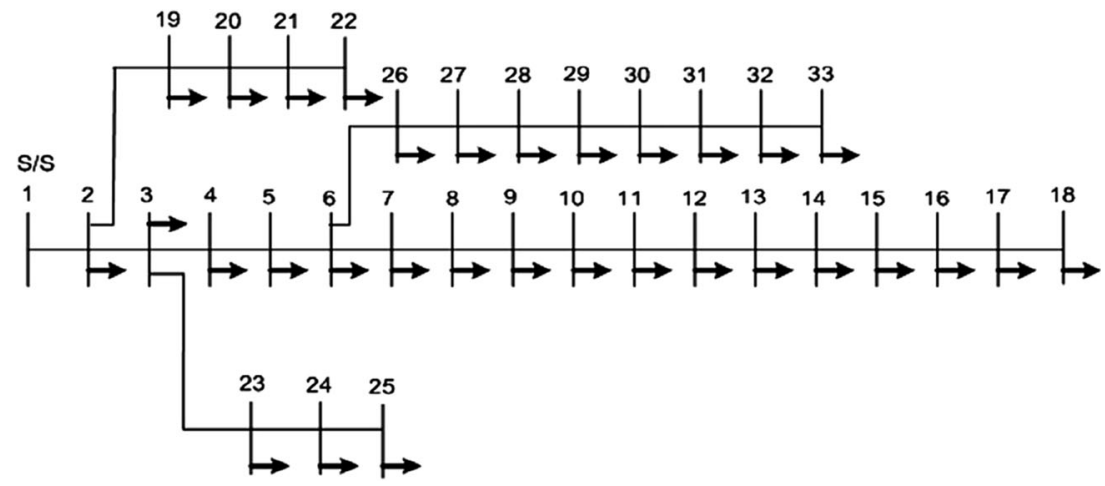

Table1 Results of DG placement in IEEE 33-bus test system

\begin{tabular}{lllllll}
\hline DG location & DG type & \multicolumn{2}{l}{ DG size } & Power Loss (kW) & Power Loss Reduction (\%) \\
\cline { 3 - 5 } & & & MW & MVar & MVA, PF \\
\hline- & - & - & - & - & 210.99 & - \\
Bus-6 & Type-1 & 3.15 & - & - & 115.28 & 45.40 \\
Bus-30 & Type-2 & - & 1.23 & - & 151.28 & 28.20 \\
Bus-6 & Type-3 & - & - & $3.02,0.8$ lead & 67.96 & 67.79 \\
Bus-6 & Type-4 & 3.15 & 1.23 & - & 198.49 & 5.90 \\
\hline
\end{tabular}

Step 1: Initialization of FA parameters including generation iteration number;

Step 2: Creation of initial population of fireflies and/or evaluation of their light intensity as an objective function;

Step 3: Creation of new population of fireflies;

Comparison of all fireflies with each other regarding the objective function value and the movement of fireflies with lower light intensity toward the fireflies with higher light intensity is done by Eq. (8). Consider that light intensity and absorption along with insect distance in each step will vary.

Step 4: Evaluation of light-intensity in new fireflies;
Step 5: Determination of the best firefly in terms of light intensity;

Step 6: If the steps iterate up to the maximum number of generations, then print the final solution; otherwise, go to step 3. Fig. 2 Depicts FA flowchart.

\section{Application of FA to the DG Placement Problem}

With regard to the increase in DG penetration into distribution networks, the goal of this research is to decrease power loss and voltage deviation (improved voltage profile) by optimum
Fig. 4 Voltage profile of IEEE 33-bus test system after installation of various DG types

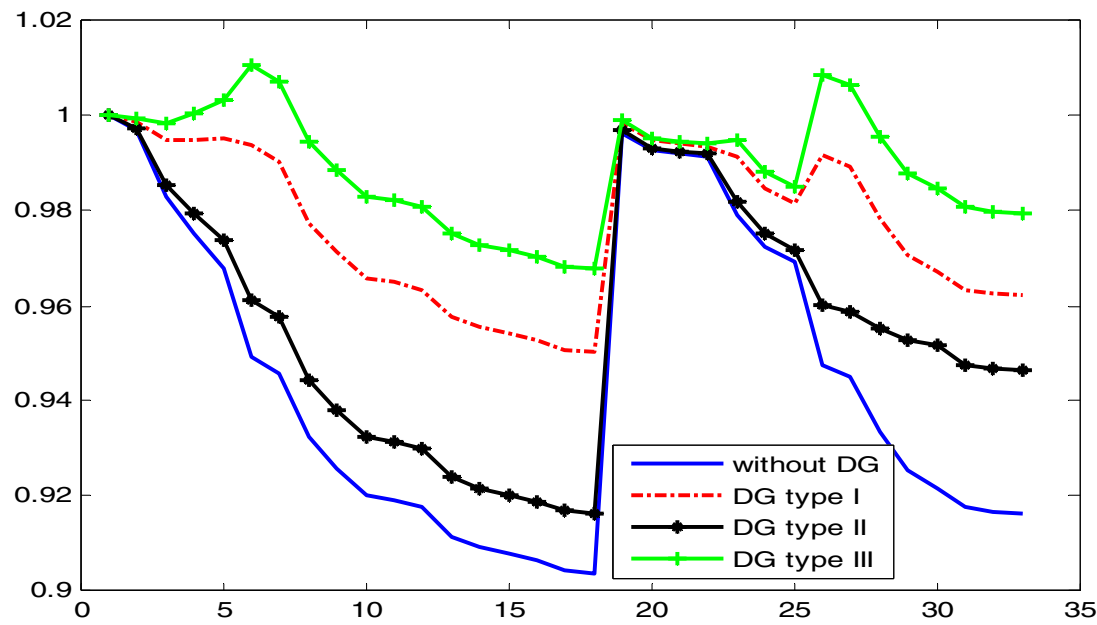


Table 2 Results without/with DGs on IEEE 69-bus test system

\begin{tabular}{|c|c|c|c|c|c|c|c|}
\hline \multirow[t]{2}{*}{ Power Loss Reduction (\%) } & \multirow[t]{2}{*}{ Power Loss (kW) } & \multicolumn{3}{|l|}{ DG size } & \multirow[t]{2}{*}{ DG type } & \multirow[t]{2}{*}{ DG location } & \multirow[t]{2}{*}{ Grid } \\
\hline & & (MVA, PF) & (MVAR) & $(\mathrm{MW})$ & & & \\
\hline- & 224.89 & - & - & - & - & - & 69buses \\
\hline 62.9 & 83.29 & - & - & 1.807 & Type1 & $61^{\text {th }}$ bus & \\
\hline 34.4 & 152.02 & - & 1.29 & - & Type2 & $61^{\text {th }}$ bus & \\
\hline 89.71 & 23.12 & $0.82,2.243$ per-phase & - & - & Type3 & $61^{\text {th }}$ bus & \\
\hline-21.4 & 273.13 & - & 1.29 & 1.807 & Type4 & $61^{\text {th }}$ bus & \\
\hline
\end{tabular}

Fig. 5 Standard IEEE 69-bus test system

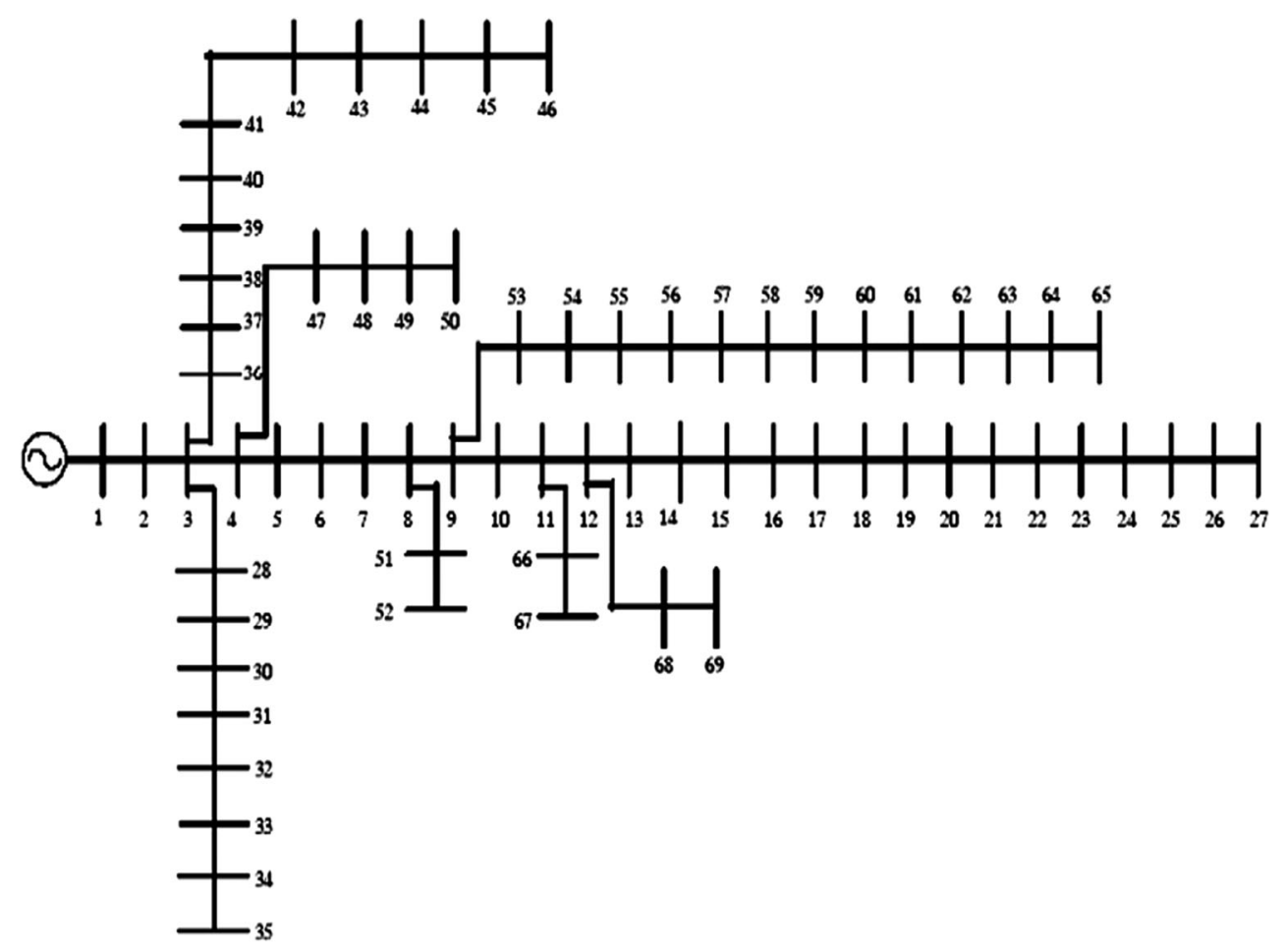

allocation of DGs. Studies have shown that improper installation of DGs (i.e., inappropriate size and location) will worsen the objectives of the DG placement compared to the state without DGs. Optimal placement of DGs can also release distribution and transmission lines capacity or defer developmental programs. Thus, new investment costs which are huge
Fig. 6 Voltage profile on IEEE 69-bus test system before/after DG placement

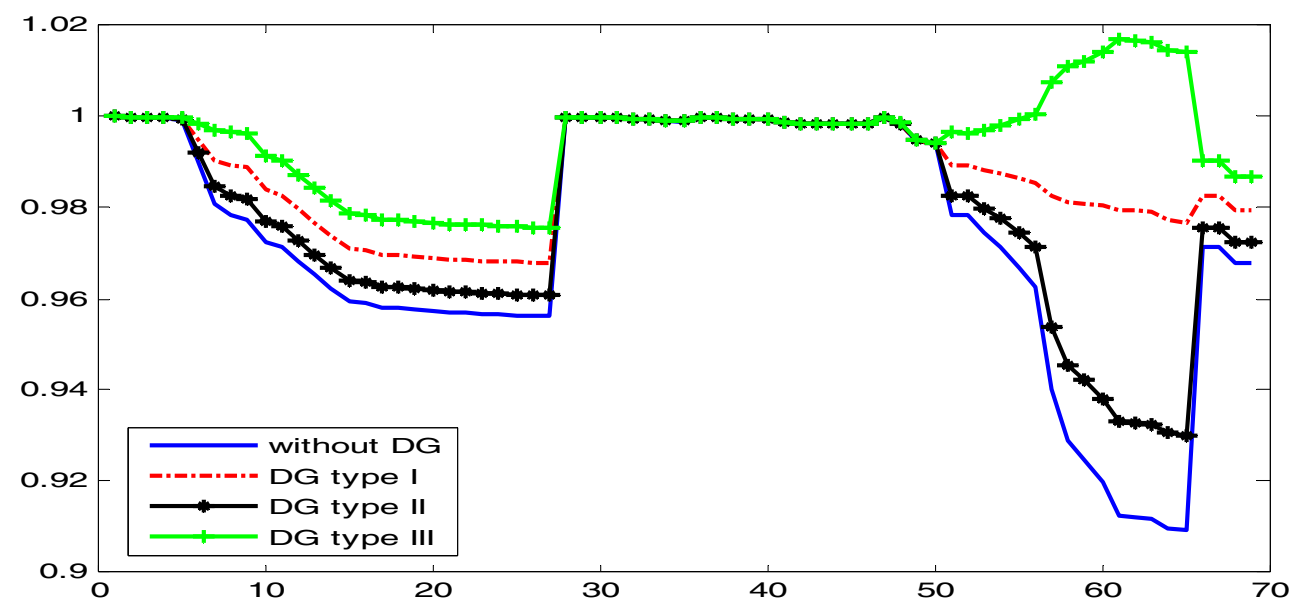


Table 3 Various types of DG placement by FA and PSO [1] in IEEE 33-bus test system

\begin{tabular}{|c|c|c|c|c|c|c|c|}
\hline \multirow[t]{2}{*}{ Method } & \multirow[t]{2}{*}{ DG location } & \multirow[t]{2}{*}{ DG type } & \multicolumn{3}{|c|}{ Optimum size of various DG types } & \multirow[t]{2}{*}{ Power Loss $(\mathrm{kW})$} & \multirow[t]{2}{*}{ Power Loss Reduction (\%) } \\
\hline & & & MW & MVar & MVA, PF & & \\
\hline Without DG & - & - & - & - & - & 210.99 & - \\
\hline \multirow[t]{4}{*}{ FA } & Bus-6 & Type-1 & 2.604 & - & - & 111.02 & 47.38 \\
\hline & Bus-30 & Type-2 & - & 1.258 & - & 151.36 & 28.26 \\
\hline & Bus-6 & Type-3 (lead) & 2.558 & 1.744 & $3.097,0.83$ lead & 67.86 & 67.84 \\
\hline & Bus-6 & Type-4 (lag) & 2.593 & 0.00003 & $2.593,1.0$ & 111.02 & 47.38 \\
\hline \multirow[t]{4}{*}{ PSO [1] } & - & - & - & - & - & 210.99 & - \\
\hline & Bus-6 & Type-1 & 3.15 & - & - & 115.29 & 45.36 \\
\hline & Bus-30 & Type-2 & - & 1.23 & - & 151.141 & 28.24 \\
\hline & Bus-6 & Type-3 (lead) & 2.47 & 1.72 & $3.02,0.82$ lead & 67.95 & 67.79 \\
\hline
\end{tabular}

Table 4 Voltage profile before and after DG type-1 installation in IEEE 33-and 69 bus test system

\begin{tabular}{lllll}
\hline \multicolumn{2}{l}{ Voltage amplitude and bus no. before DG } & & & \multicolumn{2}{l}{ Voltage amplitude and bus no. before DG } \\
\cline { 1 - 2 } Min. & Max. & & Min. & Max. \\
\hline $0.903 @ 18$ & $1.000 @ 1$ & & $0.950 @ 18$ & $1.000 @ 1$ \\
$0.909 @ 65$ & $1.000 @ 1$ & & $0.967 @ 27$ & $1.000 @ 1$ \\
\hline
\end{tabular}

and long term can be also delayed. In this paper, FA is used for power loss minimization considering exact formula for power loss as an objective function [1]. Regarding a network with $\mathrm{N}$ distribution buses, active power loss minimization is obtained by:

$P_{L}=\sum_{i=1}^{N} \sum_{j=1}^{N}\left[\alpha_{i j}\left(P_{i} P_{j}+Q_{i} Q_{j}\right)+\beta_{i j}\left(Q_{i} P_{j}-P_{i} Q_{j}\right)\right]$

where $\alpha$ and $\beta$ are provided by (7).

$$
\begin{aligned}
& \alpha_{i j}=\frac{r_{i j}}{V_{i} V_{j}} \cos \left(\delta_{i}-\delta_{j}\right) \\
& \beta_{i j}=\frac{r_{i j}}{V_{i} V_{j}} \sin \left(\delta_{i}-\delta_{j}\right) \\
& r_{i j}+j X_{i j}=Z_{i j} \\
& {\left[Z_{b u s}\right]=\left[Y_{\text {bus }}\right]^{-1}}
\end{aligned}
$$

where $P_{i}$ denotes active (real) power injected into bus $i, Q_{i}$ denotes reactive power injected into bus $i, V_{i}$ denotes voltage of bus $i, \delta_{i}$ denotes voltage angle of bus $i, Z_{i j}$ denotes an element in $i$ th row and $j$ th column of matrix $\mathrm{Z}_{\text {bus }}$, and $N$ denotes total number of network buses.
This goal is optimized considering following constraints. If technical constraints are not satisfied, the solution will not be acceptable.

- Power flow equations:

$$
\begin{gathered}
P_{G i}-P_{D i}=V_{i} \sum_{j=1}^{N} V_{j}\left(G_{i j} \cos \theta_{i j}+B_{i j} \sin \theta_{i j}\right) \\
Q_{G i}-Q_{D i}=V_{i} \sum_{j=1}^{N} V_{j}\left(G_{i j} \sin \theta_{i j}-B_{i j} \cos \theta_{i j}\right)
\end{gathered}
$$

where $\mathrm{G}_{i j}$ denotes a conductance between $i$ th bus and $j$ th bus and $B_{i j}$ denote the suceptance between $i$ th bus and $j$ th bus, respectively.

$$
\begin{aligned}
P_{i} & =P_{G i}-P_{D i} \\
Q_{i} & =Q_{G i}-Q_{D i}
\end{aligned}
$$

\begin{tabular}{|c|c|c|c|}
\hline \multicolumn{2}{|c|}{ Voltage amplitude and bus no. before DG } & \multicolumn{2}{|c|}{ Voltage amplitude and bus no. before DG } \\
\hline Min. & Max. & Min. & Max. \\
\hline $0.903 @ 18$ & $1.000 @ 1$ & $0.918 @ 18$ & $1.000 @ 1$ \\
\hline 0.909@65 & $1.000 @ 1$ & $0.930 @ 65$ & $1.000 @ 1$ \\
\hline
\end{tabular}

Where $P_{G i}$ and $Q_{G i}$ are produced powers by generators in bus $i$ and $P_{D i}$ and $Q_{D i}$ are active and reactive loads in bus $i$, respectively.
Table 5 Voltage profile before and after DG type-2 installation in IEEE 33-and 69bus test system 
Table 6 Voltage profile before and after DG type-3 installation in IEEE 33-and 69 bus test system

\begin{tabular}{lllll}
\hline \multicolumn{2}{l}{ Voltage amplitude and bus no. before DG } & & & \multicolumn{2}{l}{ Voltage amplitude and bus no. before DG } \\
\cline { 1 - 1 } Min. & Max. & & Min. & Max. \\
\hline $0.903 @ 18$ & $1.000 @ 1$ & & $0.957 @ 18$ & $1.000 @ 6$ \\
$0.909 @ 65$ & $1.000 @ 1$ & & 0.972 .27 & $1.000 @ 1$ \\
\hline
\end{tabular}

\begin{tabular}{|c|c|c|c|c|c|c|}
\hline \multirow{2}{*}{$\begin{array}{l}\text { The lowest } \\
\text { voltage }\end{array}$} & \multirow{2}{*}{$\begin{array}{l}\text { Power loss } \\
(\mathrm{kw})\end{array}$} & \multicolumn{2}{|l|}{ Size DG } & \multirow{2}{*}{$\begin{array}{l}\text { Bus } \\
\text { no. }\end{array}$} & \multirow[t]{2}{*}{ نوعن DG } & \multirow[t]{2}{*}{ network } \\
\hline & & (MVAr) & $(\mathrm{MW})$ & & & \\
\hline \multirow[t]{2}{*}{ 0.953@18 } & \multirow[t]{2}{*}{58.44} & - & 2.532 & 6 & $\begin{array}{l}\text { Type- } 1 \text {, and }-2 \\
\quad \text { (simultaneously) }\end{array}$ & \multirow[t]{3}{*}{ 33buses } \\
\hline & & 1.249 & - & 30 & \multirow[t]{2}{*}{ Type3 (Previous section) } & \\
\hline $0.958 @ 18$ & 67.66 & 1.744 & 2.558 & 6 & & \\
\hline \multirow[t]{2}{*}{$0.972 @ 27$} & \multirow[t]{2}{*}{23.17} & - & 1.828 & 61 & \multirow{2}{*}{$\begin{array}{l}\text { Type-1, and }-2 \\
\text { (simultaneously) }\end{array}$} & \multirow[t]{2}{*}{ 69buses } \\
\hline & & 1.300 & - & 62 & & \\
\hline $0.972 @ 27$ & 23.11 & 1.298 & 1.831 & 61 & Type3 (Previous section) & \\
\hline
\end{tabular}

Table 7 Simultaneous placement of DG type- 1 and type- 2 using FA

\section{- Voltage inequality constraint}

$V_{\min } \leq V_{i} \leq V_{\max } \quad i=1,2,3, \ldots, N$

\section{- Current inequality constraint}

$I_{i} \leq I_{i}^{\text {Rated }}$

where $I_{i}^{\text {Rated }}$ is acceptable current for $i$ th current with secure thermal range.

\section{Calculation Procedure}

Location and capacity of various DGs are determined by analytical method. This method is explained in detail as follows.

Consider computational formula for power loss as Eq. (6), the total power loss is minimized when partial derivative of power loss with respect to injected power is zero. That is, the change of rate of power loss to injected power is zero [1]. $\frac{\partial P_{L}}{\partial P_{i}}=2 \alpha_{i i} P_{i}+2 \sum_{\substack{j=1 \\ j \neq i}}^{N}\left(\alpha_{i j} P_{j}-\beta_{i j} Q_{j}\right)=0$

This equation is followed by:

$$
\begin{gathered}
\alpha_{i i} P_{i}-\beta_{i i} Q_{i}+\sum_{\substack{j=1 \\
j \neq i}}^{N}\left(\alpha_{i j} P_{j}-\beta_{i j} Q_{j}\right)=0 \\
P_{i}=\frac{1}{\alpha_{i i}}\left[\beta_{i i} Q_{i}+\sum_{j=1, j \neq i}^{N}\left(\alpha_{i j} P_{j}-\beta_{i j} Q_{j}\right)\right]
\end{gathered}
$$

where $P_{i}$ is injected active power in bus $i$ that is the difference between generated active power and demanded reactive power in that bus obtained by:

$P_{i}=\left(P_{D G i}-P_{D i}\right)$

where $P_{D G i}$ is injected active power by DG in bus $i$ and $P_{D i}$ is demanded load in bus $i$. by substitution pf (14) in (13), $P_{D G i}$ is obtained by:
Table 8 Simultaneous placement

\begin{tabular}{|c|c|c|c|c|c|}
\hline \multirow[t]{2}{*}{ Losses(kw) } & \multicolumn{2}{|l|}{ Size DG } & \multirow[t]{2}{*}{ Bus no. } & \multirow[t]{2}{*}{ type DG } & \multirow[t]{2}{*}{ network } \\
\hline & (MVAr) & $(\mathrm{MW})$ & & & \\
\hline 58.45 & $\begin{array}{l}- \\
1.2258\end{array}$ & $\begin{array}{l}2.5317 \\
-\end{array}$ & $\begin{array}{r}6 \\
30\end{array}$ & Type-1, and - 2 (simultaneously) & 33-bus \\
\hline 23.17 & $\begin{array}{l}- \\
1.3003\end{array}$ & $\begin{array}{l}1.8285 \\
-\end{array}$ & $\begin{array}{l}61 \\
61\end{array}$ & Type-1, and - 2 (simultaneously) & 69-bus \\
\hline
\end{tabular}
of DG type-1 and type-2 using PSO 
Fig. 7 Voltage profile of IEEE 33-bus test system after installation of various DG types

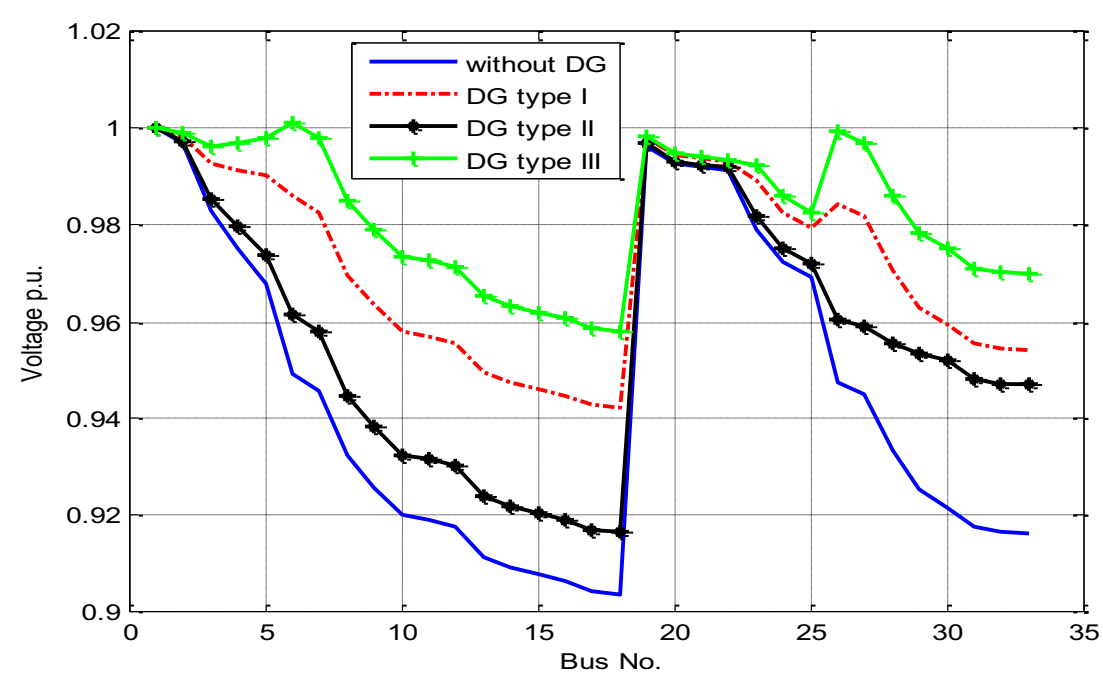

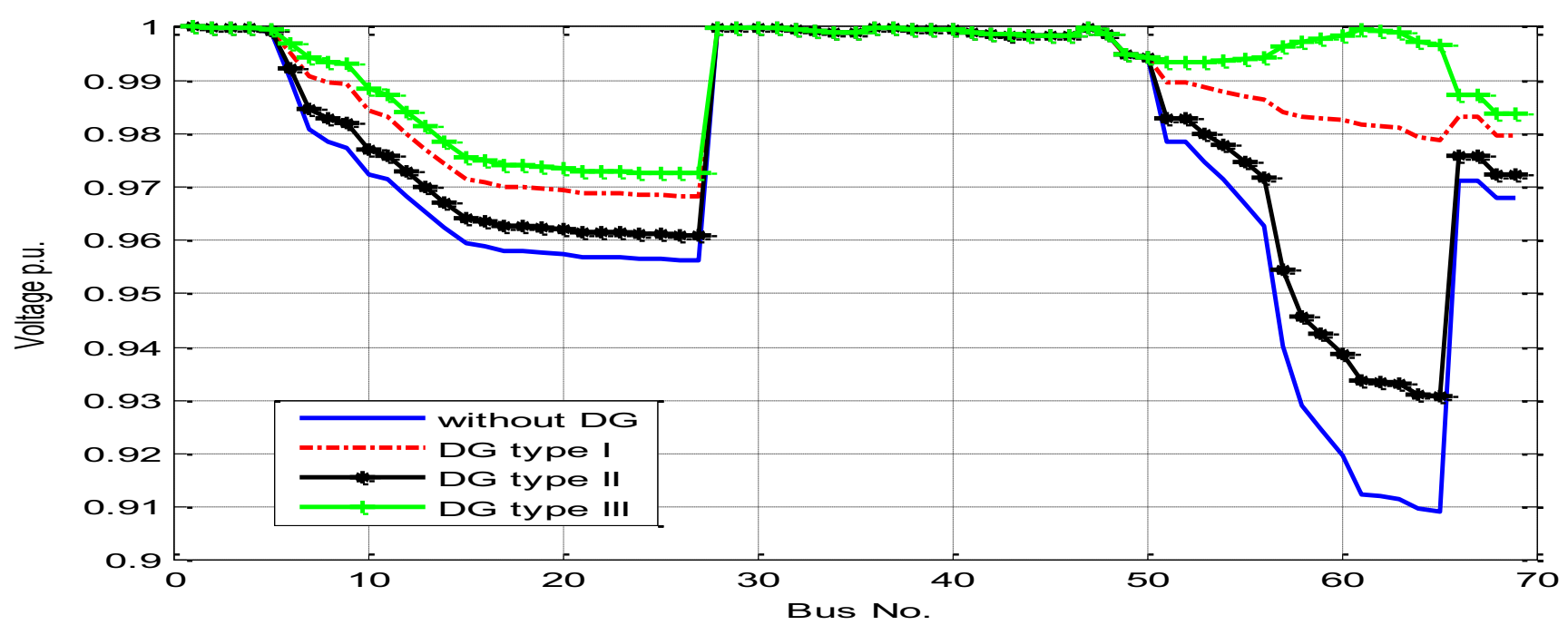

Fig. 8 Voltage profile of IEEE 69-bus test system after installation of various DG types

$P_{D G i}=P_{D i}-\frac{1}{\alpha_{i i}}\left[\sum_{\substack{j=1, j \neq i}}^{N}\left(\alpha_{i j} P_{j}-\beta_{i j} Q_{j}\right)\right]$

Similarly, for reactive power we have:

$$
\frac{\partial Q_{L}}{\partial P_{i}}=2 \alpha_{i i} Q_{i}+2 \sum_{j=1}^{N}\left(\alpha_{i j} Q_{j}-\beta_{i j} P_{j}\right)=0
$$

Table 9 Simultaneous placement of DG type-1 and type- 2 using analytical method

\begin{tabular}{|c|c|c|c|c|c|c|}
\hline \multirow[t]{2}{*}{ Losses (kw) } & \multicolumn{3}{|l|}{ Size DG } & \multirow[t]{2}{*}{ Bus no. } & \multirow[t]{2}{*}{ Type DG } & \multirow[t]{2}{*}{ Grid } \\
\hline & (MVA, pf) & (MVAr) & $(\mathrm{MW})$ & & & \\
\hline \multirow[t]{2}{*}{58.48} & - & - & 2.490 & 6 & \multirow[t]{2}{*}{ Type-1, and - 2 (simultaneously) } & \multirow[t]{2}{*}{33 bus } \\
\hline & - & 1.23 & - & 30 & & \\
\hline 67.94 & $3.02,0.08$ & - & - & 6 & Type-3 & \\
\hline \multirow[t]{2}{*}{23.13} & - & - & 1.807 & 61 & \multirow[t]{2}{*}{ Type-1, and -2 (simultaneously) } & \multirow[t]{2}{*}{ 69bus } \\
\hline & - & 1.29 & - & 61 & & \\
\hline 23.13 & $2.22,0.81$ & - & - & 61 & Type-3 & \\
\hline
\end{tabular}


Fig. 9 Voltage profile of IEEE 33-bus test system after simultaneous placement of DG type-1 and type- 2

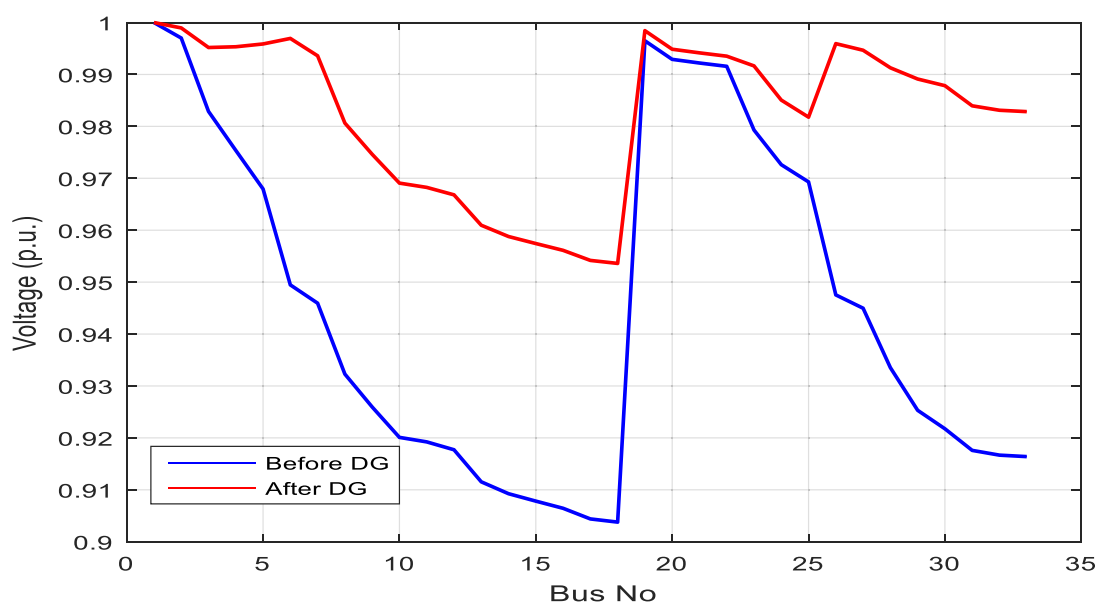

Where

$Q_{i}=\left(Q_{D G i}-Q_{D i}\right)$

Thus,

$Q_{D G i}=Q_{D i}-\frac{1}{\alpha_{i i}}\left[\sum_{\substack{j=1 \\ j \neq i}}^{N}\left(\alpha_{i j} Q_{j}+\beta_{i j} P_{j}\right)\right]$

Eq. (15) represents DG type-1 and Eq. (18) represents DG type- 2 in each bus [1]. If DG type-1 placed on bus $i$ leads to power loss less than that of DGs placed on each bus, $i$ th bus is selected as the optimum place for DG type-1 installation. Similarly, if DG type-2 placed on $j$ th bus results in power loss lower than that of DGs placed on each bus, $j$ th bus is considered as the optimum position for DG type- 2 installation. Regarding placement of DGs of type-1 and type-2, Eqs. (15) and (18) would be combined with DG type-3 in ith bus to determine size and power factor of DG. Considering any capacities for DGs higher than $P_{D G i}$ and $Q_{D G i}$ in $i$ th bus leads to higher power loss. Power factor of DG type-3 is given by Eq. (19):
$O P F=\frac{P_{D G i}}{\sqrt{P_{D G i}^{2}+Q_{D G i}^{2}}}$

If optimum positions for DG type- 1 and type- 2 are different on bus $i$ and bus $j$, respectively, DG type- 3 may be installed on bus $i$ with calculated active and reactive powers by Eqs. (15) and (18). Power factor of DG type-3 for this condition is determined by Eq. (19).

In this research, DG type- 3 is considered for active and reactive power generation. Regarding load with phase-lead power factor, DG with a capability of absorbing reactive power is required.

A bus with the minimum power loss is considered as the optimum position for DG installation. Computational procedure to find optimum place and capacity of DGs are explained by [1]:

Step 1: Run power flow for base network (without installation of DGs);

Step 2: Calculate power loss by Eq. (5);

Step 3: Get various DGs size on each bus except reference bus using Eqs. (15) and (18) to achieve minimum active power.
Fig. 10 Voltage profile of IEEE 69-bus test system after simultaneous installation of type- 1 and -2 DGs

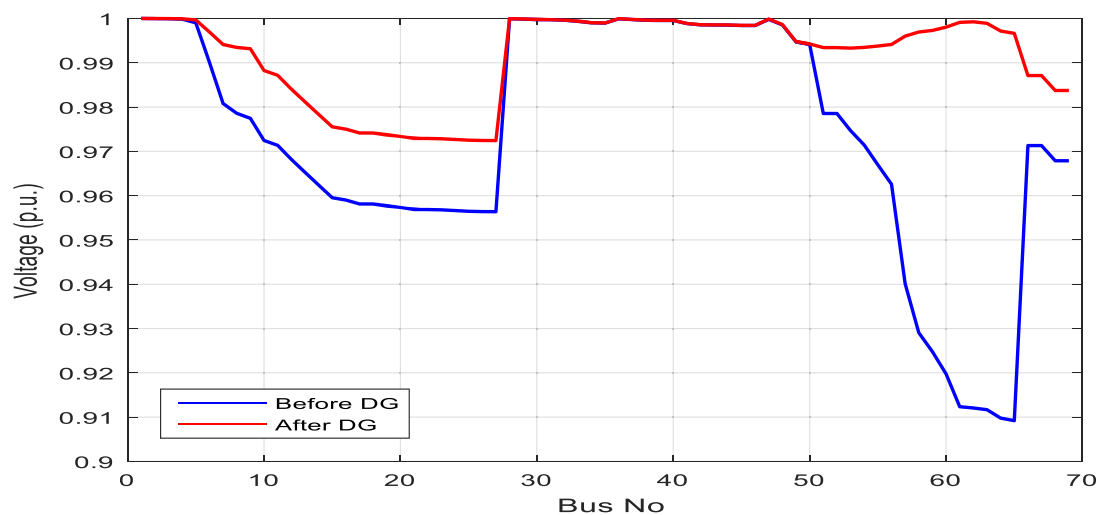


Fig. 11 Size of DG type-3 in IEEE 33-bus test system by analytical method

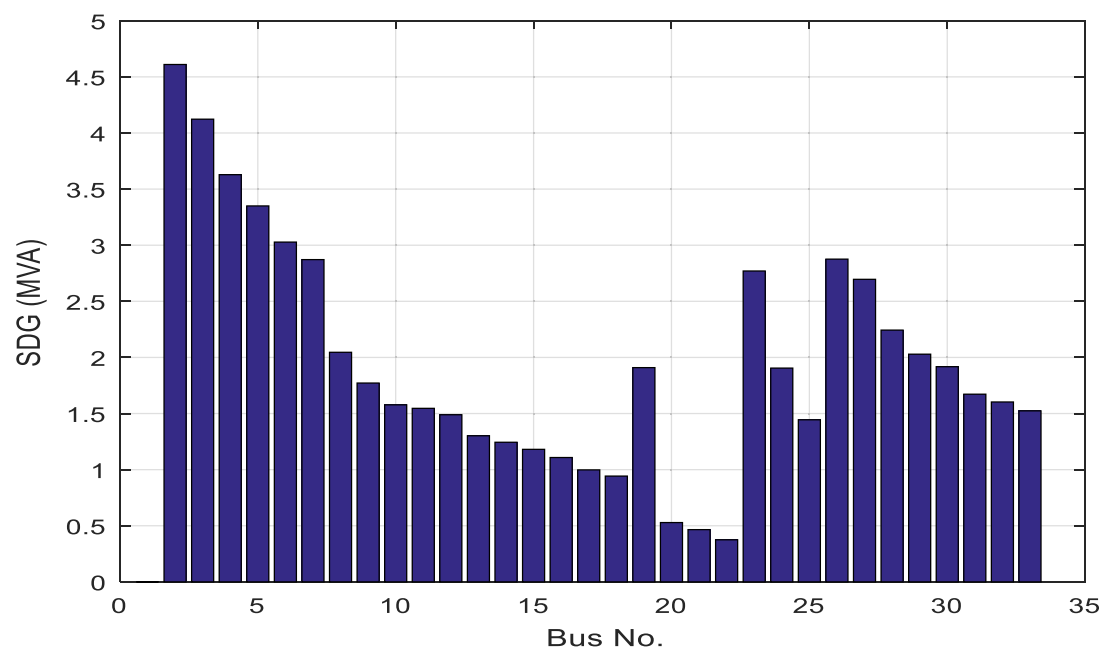

Step 4: Consider possible constraint violation after DG installation and employ it in step 3 for each bus.

Step 5: A bus with minimum power loss is considered as the optimum place (while all constraints are satisfied);

Step 6: Calculate optimum power factor using Eq. (19) for DG type-3;

Step 7: Run power flow program with determined optimum size of DG in optimum bus.

Step 8: Calculate power loss after DG placement.

Above equation gives optimum DG size in order to have minimum power loss for each bus $i$. Any capacities except $P_{D G i}$ installed in bus $i$ leads to increased power loss. This power loss is a function of $\alpha$ and $\beta$. When DG is installed in the network, power loss coefficients vary because they are dependent on voltage and angle parameters. Updating $\alpha$ and $\beta$ requires reexecution of power flow program.

\section{Case Study (1)}

Standard IEEE 33-bus test system is illustrated in Fig. 3 Data of IEEE 33-bus test system [18]. This test system has the following power rate: active power of $3.715 \mathrm{MW}$ and reactive power of 2.300 MVar with voltage ratings of $12.66 \mathrm{kV}$. Data of active and reactive power along with lines impedance is provided in Table 1. in this section, the effect of DGs on IEEE 33-bus test system is evaluated.

Network active power loss is $210.99 \mathrm{~kW}$, the minimum voltage amplitude is in bus 18 (0.90378 p.u.).

Table II provides the effect of various DG types installed on IEEE 33-bus test system. Voltage profile of the test system is shown in Fig. 4. Obviously, installation of DG type-3 on bus-6 leads to the best voltage profile. In addition, as seen in Table 2, DG type- 3 installation on bus- 6 has the most recued power loss. Obtained results are consistent with Refs. [ [1 and 19]]. One DG type- 4 is installed on bus- 6 with active and reactive
Fig. 12 Network power loss in the presence of DG type-3 with various sizes in IEEE 33-bus test system

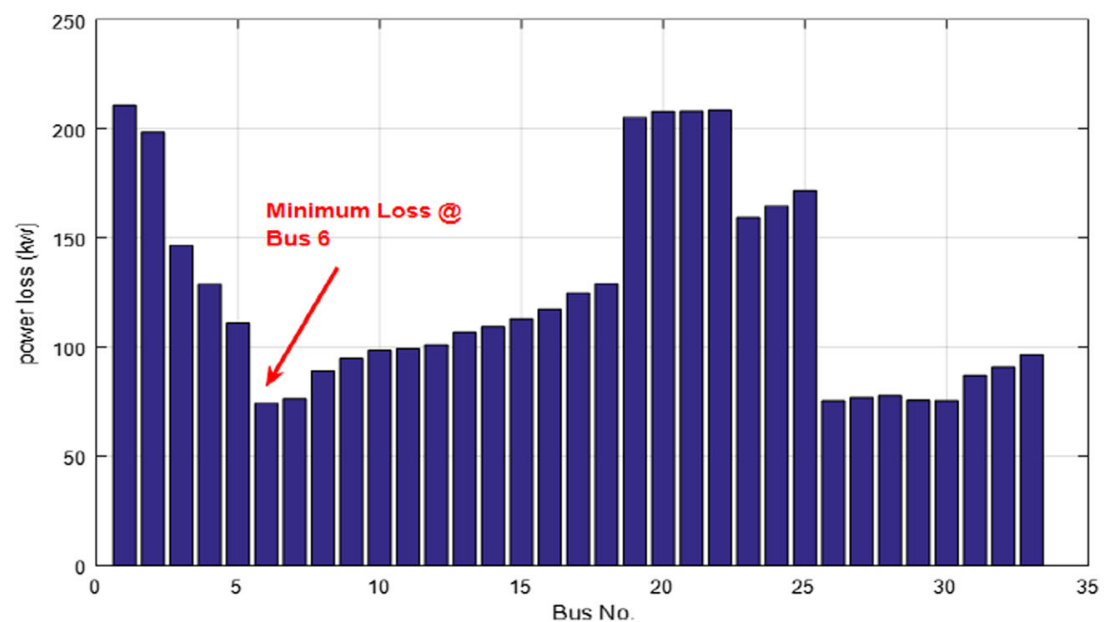


Fig. 13 Size of DG type-1 and type-2 in IEEE 33-bus test system by analytical method

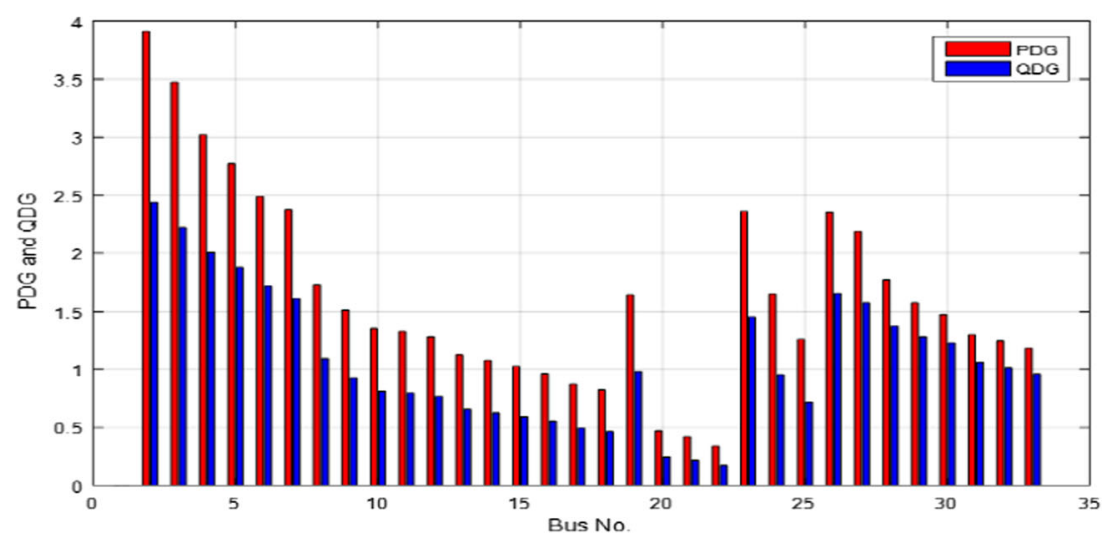

powers of 3.15 MW and 1.23 MVar, respectively. As seen, power loss is improved slightly; however, it has the worst results among the other states.

\section{Case Study (2)}

One-line diagram of IEEE 69-bus test system is illustrated in Fig. 5. The related data are given in Table 2 that is taken from Ref. [18].

The active power loss was $224.89 \mathrm{~kW}$, the lowest voltage is in bus-65 (0.9092 p.u.).Fig. 6

Table 2 gives the results of DG placement on IEEE 69-bust test system. In addition, Fig. 2 shows voltage profile for this test system with different DG types. Obviously, Type-3 DG installation on bus-61 leads to the best results. Further, as seen in Table 2, the presence of type-3 DG on bus-69 results on more reduction in power loss. The obtained results are in harmony with those in [10]. Simulation results for simulation of type-4 DG on bus-61 are provided in Table 2 with active and reactive powers of $1.8078 \mathrm{MW}$ and 1.29 MVAr, respectively. As observed, the power loss raises, highlighting that the reactive power consumption by DG has unfavorable impact on power loss in distribution system.

\section{Results and Discussion}

FA is tested on two standard test systems. In this section, optimum site and size of various DG types (i.e., Type-1, Type-2 and Type-3) are obtained by FA and listed in Table 3. Obtained results by PSO are also given in Table 4 . As seen in this table, placing DG type-1 leads to power loss reduction to $111 \mathrm{~kW}$, while this value is $151 \mathrm{~kW}$ by DG type2 , and the power loss is only $68 \mathrm{~kW}$ by DG type-3. Additionally, optimization is done for DG type-4. According to this table, the optimal state in terms of power loss reduction is obtained when reactive power of the units is zero. Here, this value is $0.00003 \mathrm{MVar}(\cong 0)$. Comparison of DG placement of type-1, -2 , and -3 in two IEEE 33 -and 69 bus test systems by FA and PSO are provided in Table 2 and 3. The results reveal that FA is able to reach less power loss reduction with less DG size compared to PSO. Furthermore, it is seen that in all states considered, FA outperforms PSO.

Figure 5 depicts voltage profile of IEEE 33-bus test system when various DG types are installed. It is seen that installing DG type-3 is the most advantageous among all states in voltage profile.

Investigation of results given in Table 3 and 4 demonstrated that DGs that inject active and reactive powers into the
Fig. 14 Power loss in the presence of DG type-1 and type-2 with various sizes in IEEE 33-bus test system




Fig. 15 Type-3 DG size on IEEE 69-bus test system obtained by analytic method
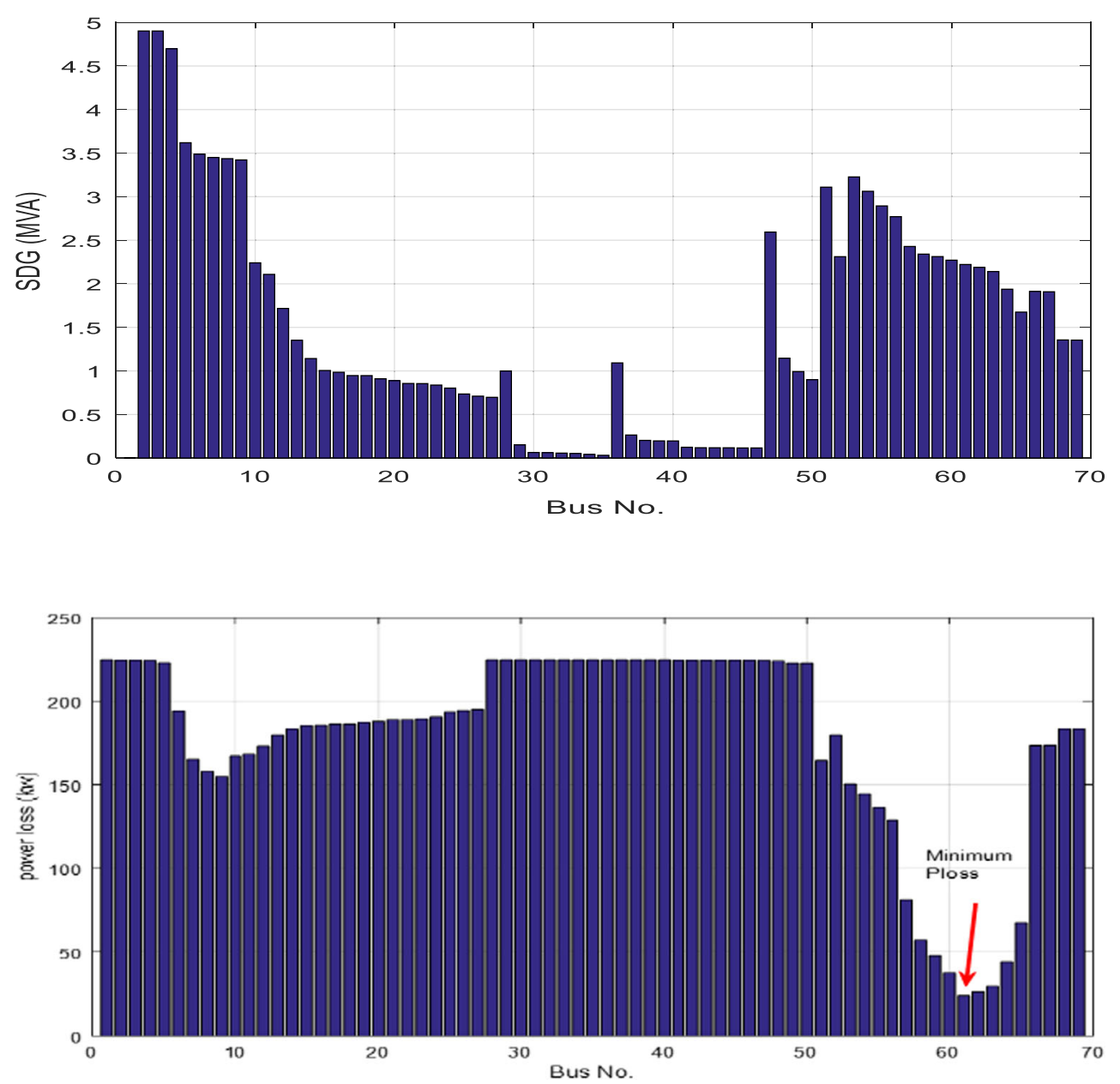

Fig. 16 Power loss on IEEE 69bus test system in the presence of various type-3 DGs network lead to more power loss reduction compared to those that inject only active power. Tables 4 and 5 provide minimum and maximum voltages for type-1, -2, and - 3 in IEEE 33-69 bus test system.

Considering the results in the tables, voltage profile is improved remarkably in all states after DG placement. Overall, voltage profile improvement in DG type- 3 is the best among all. Table 6

\section{Simultaneous Placement of DG Type- 1 and -2 by FA}

If two DGs of type-1 and type- 2 are place in simultaneous manner, power loss may be reduced up to the case when a DG type- 3 is installed. Obtained results when FA and PSO are employed for this case are given in Tables 7 and 8 .

Voltage profile related to IEEE 33-, and 69-bus test systems before and after Type-1, and-2 DG installation in
Fig. 17 Type-1, -2 DG size on IEEE 69-bus test system obtained by analytic method

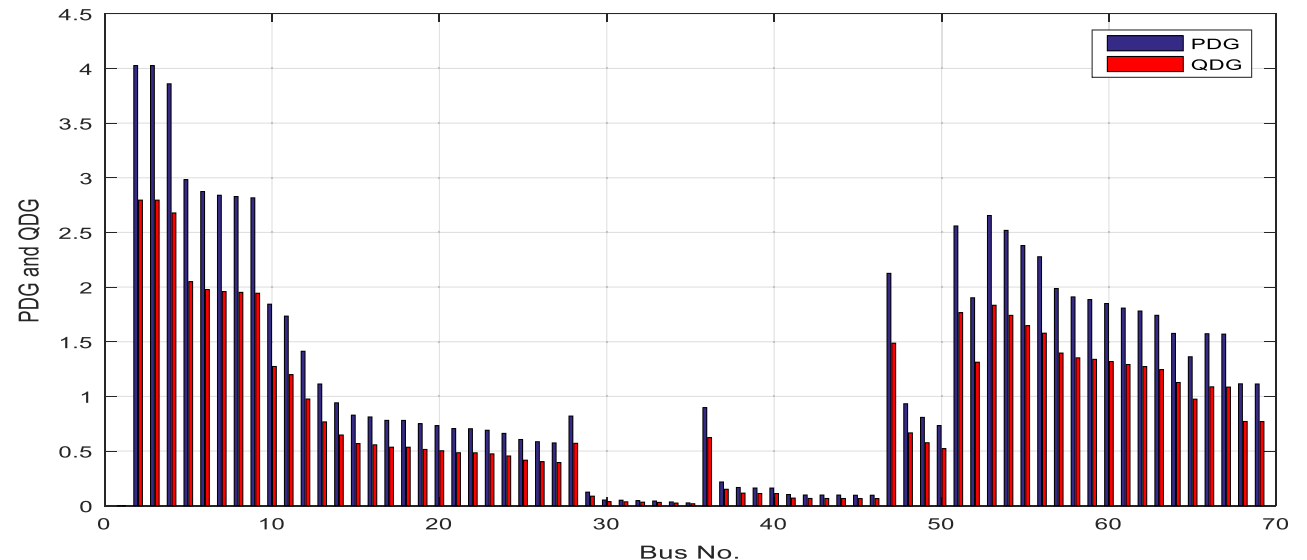


Fig. 18 Power loss on IEEE 69bus test system in the presence of various type-1, -2 DGs

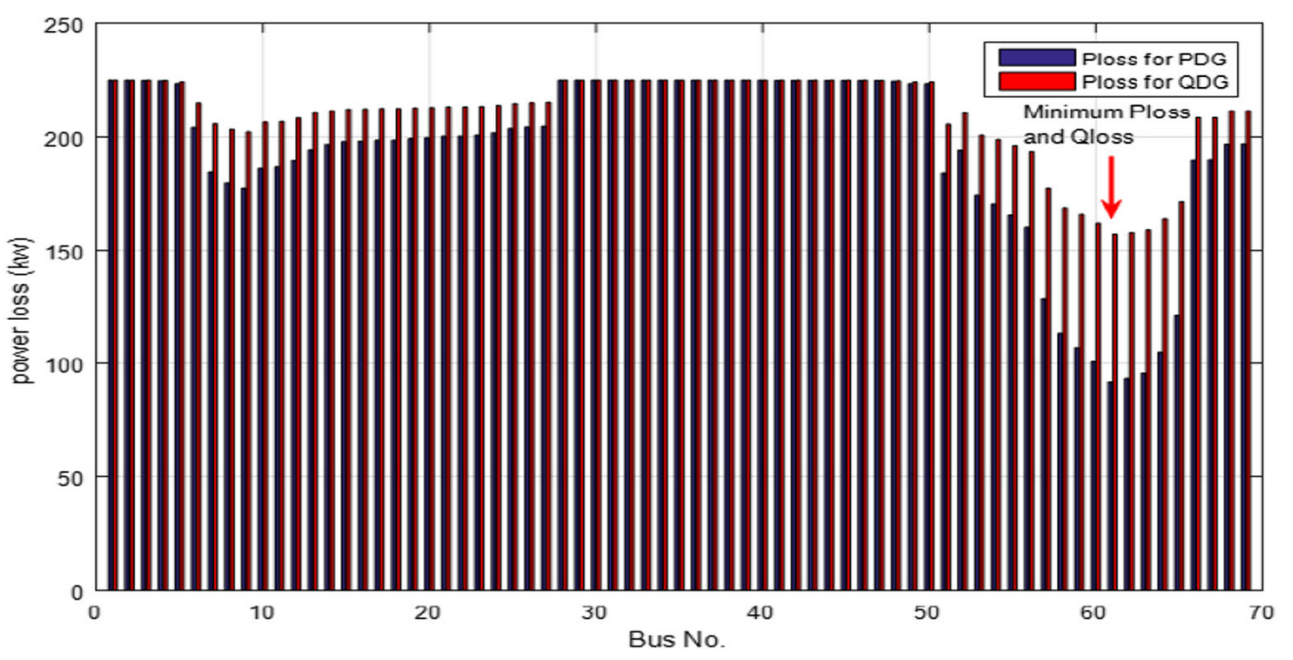

simultaneous manner by FA is depicted in Figs. 7, and 8, respectively. Comparing the results of type-1 and -2 DG installation with those of type-3 DG installation reveals that the former is better in terms of power loss reduction in IEEE 33bus test system; however, the results in 66-bus test system are similar.

\section{Placement with Analytical Method}

According to Table 9, placement of DG type-1 and type-2 through analytical method reaches similar results as FA. Evidently, when a DG of type- 1 and type- 2 is placed in optimum places (type-1 @ bus 6 and type-2 @ bus 30), active power losses is reduced from $67.95 \mathrm{~kW}$ (type-3) to $58.45 \mathrm{~kW}$. And, the results of DG type-3 is slightly different from FA. For IEEE 33-bus test system, the size of DG type-3 obtained by FA is $3.09 \mathrm{MW}$; while, this is $3.07 \mathrm{MW}$ by analytical method. In addition, the obtained power factor is the same in both cases. In IEEE 69-bus test system, optimum size (i.e., 2.245 MVA, 0.82 lead) for type-3 DG was obtained using FA; while, 2.222 MVA, 0.81 lead phase was obtained via analytic method. The obtained results with these two methods are very close to each other.

In analytical method, the size of a DG type- 1 and type- 2 for each bus is achieved for power loss minimization. For larger networks, it is possible that this method is time-consuming for placement of DG type-1 and type-2. Analytical method for multiple DG placement is not appropriate to reduce power loss. Thus, intelligent methods to determines the optimum size and site of multiple DGs in larger networks. Figs. 9 and 10 depict the DG size for type-3 in various buses; while Figs. 11 and 12 illustrate power loss in the presence of DG type- 3 with various sizes in IEEE 33-in69 bus test system. Considering that selection of proper place and capacity for DG installation is done based on minimum power loss, the bus with a DG its size is obtained leads to minimum power loss (highlighted by arrows).
Figures 13, 14, 15, and 16 are for IEEE 69-bus test system.

\section{Conclusion}

The use of DGs in distribution networks has remarkable advantages such as network power loss reduction and voltage profile improvement in distribution system. Fig. 17 In this paper, placement of various DGs is studied in terms of active power loss in distribution networks. Results obtained in the experiments reveal that application of DGs greatly influences power loss. In addition, improvement value that is appeared on power loss and voltage profile is dependent on installation place and capacity of the DG units. Considering that power loss reduction is an important index not only in operation but also in network design, it is considered as the objective function for the optimization problem. Firefly algorithm is considered as optimization tool in this research. Fig. 18 Validity of the power flow results are completed on IEEE 33-in 69 bus test system. In addition, results obtained by this optimization algorithm on test system revealed that FA is highly efficient.

\section{References}

1. Mohanty B, Tripathy S (2016) A teaching learning based optimization technique for optimal location and size of DG in distribution network. J Electr Syst Inf Technol 3(1):33-44

2. Hung DQ, Mithulananthan N, Bansal RC (2013) Multiple distributed generators placement in primary distribution networks for loss reduction. IEEE Trans Ind Electron 60(4):1700-1708

3. Ali ES, Abd Elazim SM, Abdelaziz AY (2018) Optimal allocation and sizing of renewable distributed generation using ant lion optimization algorithm. Electr Eng 100:99-109. https://doi.org/10. 1007/s00202-016-0477-z

4. Imran Ahmad Quadri*, S. Bhowmick, D. Joshi, A comprehensive technique for optimal allocation of distributed energy resources in radial distribution systems, Appl Energy 211 (2018) 1245-1260 
5. Niloofar Ghanbari, "Optimizing operation indices considering different types of distributed generation in microgrid applications," received: 15 march 2018; accepted: 8 April 2018; published: 11 April 2018,MDPI

6. FahadIqbal,, Optimal placement of DG and DSTATCOM for loss reduction and voltage profile improvement, Alexandria Engineering Journal Volume 57, Issue 2, June 2018, Pages 755-765

7. Godha (Dagade) N.R., Bapat V.N., Korachagaon I. (2020) Placement of distributed generation in distribution networks: a survey on different heuristic methods. In: Pawar P., Ronge B., Balasubramaniam R., Vibhute A., Apte S. (eds) Techno-societal 2018. Springer, Cham-07 November 2019

8. Sujatha M.S., Roja V., Nageswara Prasad T. (2019) Multiple DG placement and sizing in radial distribution system using genetic algorithm and particle swarm optimization. In: computational intelligence and big data analytics. SpringerBriefs in applied sciences and technology. Springer, Singapore-09 September 2018

9. Moradi MH, Abedini M (2012) A combination of genetic algorithm and particle swarm optimization for optimal DG location and sizing in distribution systems. Electr Power Energy Syst:66-74

10. Bhumkittipich K, Phuangpornpitak W (2013) Optimal placement and sizing of distributed generation for power loss reduction using particle swarm optimization. Energy Procedia:307-317

11. Bagheri AA, Habibzadeh A, Alizadeh SM (2011) Comparison of the effect of combination of different DG types on loss reduction using APSO algorithm. Canadian Journal on Electrical and Electronics Engineering 2(10):468-474

12. Moradi MH, Reza Tousi SM, Abedini M (2014) Multi-objective PFDE algorithm for solving the optimal siting and sizing problem of multiple DG sources. Electr Power Energy Syst:117-126
13. Ebrahimian H Optimal operation of energy at hydrothermal power plants by simultaneous minimization of pollution and costs using improved ABC algorithm. Frontiers in Energy 9(4):426-432

14. Chen TH, Sh. Chen M, Hwang KJ, Kotas P, Chebli EA (1991) Distribution system power flow analysis - a rigid approach. IEEE Transaction on Power Delivery 6(3):1146-1152

15. Eminoghlu U, Hocaoglu MH (2005) A new power flow method for radial distribution systems including voltage dependent load models. Electr Power Syst Res 76:106-114

16. A Siadatan, P Farhadi, Optimal Placement of Various Distributed Generations in Distribution Systems using Firefly Algorithm,, 2018 4th International Conference on Electrical Energy Systems (ICEES) IEEE

17. Alireza Siadatan, Bahman Taheri, Mahsa Sedaghat, New fundamental modulation technique with SHE using shuffled frog leaping algorithm for multilevel inverters, Evolving Systems, Springer Berlin Heidelberg 2019

18. Manafi H, Ghadimi N, Ojaroudi M (2013) P Farhadi. Optimal placement of distributed generations in radial distribution systems using various PSO and DE algorithms Elektronika ir Elektrotechnika 19(10):53-57

19. Davar Mirabbasi, Bahman Taheri, Optimal Reconfiguration of Intelligent Distribution Net-works in the Presence of DGs using TLBO Algorithm,, Vol 7 No 3 (2018): September, Majlesi Journal of Energy Management

Publisher's Note Springer Nature remains neutral with regard to jurisdictional claims in published maps and institutional affiliations. 